\title{
Color from Shape from Color: A Simple Formalism with Known Light Sources
}

\author{
Mark S. Drew Michael H. Brill \\ School of Computing Science, Sarnoff Corporation, \\ Simon Fraser University, 201 Washington Rd., \\ Vancouver, B.C., $\quad$ Princeton, NJ \\ Canada V5A 1S6 U.S.A 08540 \\ mark@cs.sfu.ca_mbrill@sarnoff.com
}

\author{
Mailing address: \\ Dr. Mark S. Drew \\ School of Computing Science, \\ Simon Fraser University, \\ Vancouver, B.C., \\ Canada V5A 1 S6 \\ Telephone: 604-291-4682 \\ Fax: 604-291-3045
}

(C)M.S. Drew, M.H. Brill 2000

http://fas.sfu.ca/cs/people/Faculty/Drew/index.html 


\begin{abstract}
Photometric stereo is a well-known technique for recovering surface normals of a surface but requires three or more images of a surface taken under illumination from different directions. At best, one may dispense with the need for multiple images by using colored lights tuned to camera filters. But a less restrictive paradigm is available using the Orientation-from-Color approach, wherein multiple broadband illuminants impinge on a surface simultaneously. In that method, colors for a Lambertian surface lie on an ellipsoid in color space. The method has mostly been applied to single-color objects, with ellipsoid quadratic form parameters determined from a large number of pixels. However, recently Petrov et al. developed an entirely local approach, useful also for multicolored objects with color uniform in each patch. Here we investigate to what extent a method like Petrov's can be applied in the ostensibly simpler situation in which the complex lighting environment is known, i.e. a color photometric stereo situation, with all lights at play at once with only a single image to analyze. We find that, assuming a simple model of color formation, we are able to recover the object colors along with surface normals, using only a single image. Because we immerse the object in a known lighting environment, we show that only half of the equations utilized by Petrov are actually needed, making the method more stable. Nevertheless solutions do not exist at every pixel; instead we may determine a best estimate of patch color using a robust estimator, and then apply that estimate throughout a patch. Results are shown to be quite good, compared to ground truth. The simple color model can often be made to hold more exactly by transforming the color space to one corresponding to spectrally sharpened sensors, which are a matrix transform away from the actual camera sensors. Here, the reliability and accuracy of the normal vector and surface color recovery algorithm is improved by this straightforward transformation.
\end{abstract}

OCIS numbers: 150.0150 Machine vision, 330.1720 Color vision, 100.2960 Image analysis, 100.3190 Inverse

problems, 999.9999 Shape from Shading. 


\section{Introduction}

Image formation under simultaneous illumination by a set of colored lights implies that under fairly general conditions Lambertian surfaces with uniform color have RGB pixel values $\rho$ that are a linear transform away from surface normals $[1,2,3,4,5,6,7,8,9]$. Then $\rho$ values lie on a quadratic form in color space. Previously, emphasis has been on recovering the linear transform matrix relating lighting and surface normals, under unknown lighting conditions, for uniformly-colored surfaces. Petrov and Antonova [10] recently extended the paradigm examined by considering a surface made up of uniformly-colored curved patches, under complex lighting. This is the situation we wish to investigate here. (The situation of many colored patches under simple lighting was investigated in [11].)

Petrov and Antonova show how to locally recover the quadratic form in color space on which a pixel's RGB triple lies; however, actually recovering surface normals themselves requires several more steps in a complex algorithm. As in [4], their method for finding actual surface normals amounts to color image segmentation, collecting RGB information in a patch, and then minimizing an integrability-error measure, using a type of simulated annealing algorithm. This last step is necessary because although they recover the quadratic form for a color patch, they must still derive a rotation to bring recovered surface normals into the correct orientation with respect to the camera plane. While the equations they derive can indeed be used to locally compute the quadratic form matrix, here we do not need the complicated rotation-recovery step and can make use of fewer equations by employing known lighting. We also demonstrate that a simple color model allows the recovery of surface colors once surface normals are determined, and in fact using more equations does not help for this additional task.

Petrov and Antonova did not investigate what can be gleaned from a known lighting situation. Here we mean to extend the usual, known-lighting, photometric stereo (PMS) paradigm to this many-simultaneouslights situation and prove in principal that we can recover an approximation of surface normals and surface colors from a single input image. This is accomplished by the simple assumption that color consists of the lighting color multiplied component-wise times the surface color. We also find that this color model can often be made more accurate by a matrix transform of camera sensors into a another, "spectrally sharpened" set of sensors [12]

Note that while it is feasible to carry out a PMS experiment using tuned, multiplexed narrowband lights and sensors [13], in essence PMS uses three images under three different lights. Here we use many broadband lights (at least three) and broadband sensors, with all lights at once creating a single image. We are interested in determining surface normal and color information from a calibrated lighting environment and will use a very simple calibration strategy. We find that with calibrated lighting, PMS can proceed in color. The solution uses the orientation-from-color linear equations relating surface normal with color $[1,2,3,4,5,6]$, as well as the first partial derivatives of these equations [10]. In contradistinction to [10], we show that it is not necessary to further use second partial derivative equations for calculating a solution. The solution consists of a quartic equation in one color component, which may be disambiguated by either ruling out negative values in the other color components, by ruling out color values that lead to image pixel values that are physically impossible, or finally by appealing to consistency of the derivative of the solution of the first-derivative equation.

Results are shown to be quite good, both for recovered surface normals and for colors. While color may not be recoverable at every pixel in each color patch, as long as it is possible to carry out a reasonably accurate color image segmentation an approximation of the color can be determined by forming a representative of all colors recovered in a patch. Here, we use a technique from robust statistics to find the best representative color in each patch - we use the Least Median of Squares (LMS) algorithm to robustly characterize the value of the mode of the distribution for each color component in a patch. Then if that color value is extended throughout 
the patch one may recover the surface normal at every patch location, not just those with a significant color solution.

In $\S 2$ we recapitulate how complex lighting leads to a linear relationship between surface normal and color. A cognate paradigm was recently used by Healy et al. [14] wherein an object consisting of flat patches is imaged twice, once under simple lighting and then again under complex, colored lighting. Healy et al. then use the ratio image of these two to detect surface normal discontinuities. For completeness that method for surface normal segmentation is reviewed in $\$ 2.2$, in the context of a diagonal model of illumination change. One argument in favor of a diagonal model is the Factor Model of color, wherein color is taken to be the product of colors for surface and for illuminant. That model is set out in $\S 2.3$. Then using the Factor Model, in $\S 3$ it is shown how the color PMS equations specialize when such a simple color model is used. Section 4 shows how it is possible to solve for color locally in a color PMS situation (with most solution statements relegated to Appendix A). In $\S 5$ it is shown how accuracy of the color model can often be increased by spectrally sharpening the camera sensors. Experimental results to demonstrate that the method does accomplish its goals are given in $\S 6$. In $\S 7$ the geometrical meaning of the color PMS equations is outlined, and $\S 8$ contains some reflections on alternative strategies.

\section{Color Image Formation}

\subsection{Linear Transform from Normal to Color}

Consider the RGB triple $\rho$ resulting from some set of lights with spectral power distributions $I^{i}(\lambda)$ and indexed by $i=1$..L. Suppose all these illuminants impinge on a Lambertian surface with surface spectral reflectance function $S_{x}(\lambda)$, under conditions of orthography. If the camera system color sensors have sensitivity functions $\boldsymbol{q}(\lambda)$ then

$$
\boldsymbol{\rho}_{x}=\sum_{i=1}^{L}\left(\left(\boldsymbol{a}^{i}\right)^{T} \boldsymbol{n}_{x}\right) \int I^{i}(\lambda) S_{x}(\lambda) \boldsymbol{q}(\lambda) d \lambda
$$

where $\boldsymbol{n}_{x}$ is the surface normal for that pixel, indexed by 2D retinal coordinates $\boldsymbol{x}$, and light $I^{i}$ has normalized spatial direction $\boldsymbol{a}^{i}$. Eq. (1) holds if a surface point sees all lights.

Consider a particular patch with uniform surface spectral reflectance $S(\lambda)$. We note that the color $\boldsymbol{b}^{i}$ for light $I^{i}(\lambda)$ reflected from surface $S(\lambda)$ is given by

$$
\boldsymbol{b}^{i} \equiv \int I^{i}(\lambda) S(\lambda) \boldsymbol{q}(\lambda) d \lambda
$$

Thus the basic equation (1) can be rewritten in the simple form $[3,6]$

$$
\rho=B A n
$$

where we stack all the directions $\boldsymbol{a}^{i}$ row-wise into an $L \times 3$ matrix $\boldsymbol{A}$ and group the color space vectors $\boldsymbol{b}^{i}$ column-wise into a $3 \times L$ matrix $B$ :

$$
\boldsymbol{A}=\left(\boldsymbol{a}^{1}, \boldsymbol{a}^{2}, \ldots \boldsymbol{a}^{L}\right)^{T} \quad, \boldsymbol{B}=\left(\boldsymbol{b}^{1}, \boldsymbol{b}^{2}, \ldots \boldsymbol{b}^{L}\right) .
$$

Let

$$
F \equiv B A
$$


so that (3) becomes simply

$$
\rho=F n .
$$

Thus a linear transform relates color to surface normal. The model breaks down when not every illuminant can be seen from a particular surface patch, so that $F$ is not the same there as at a patch illuminated by all lights. This is the case when the surface is in shadow or is self-shadowed or has specularities or non-uniform color.

For completeness, in section $\S 2.2$ below we recapitulate how approximations of the full model can be used to express illumination change via a diagonal model, and how such an approximation has been used to recover surface normal information for surfaces with planar flat areas with nonuniform color. But again, here we do not wish to change the illumination, and in fact we focus on curved surfaces that have piecewise-uniform patches of color.

\subsection{Diagonal Model of Illumination Change}

In [14] two different lighting environments are used, each in turn lighting the same scene, to segment surface normals for planar patches. The idea is that a diagonal model sufficiently well captures lighting change, so that a ratio image apprehends the lighting change. Then segmentation of the ratio image also segments planar patch normal changes.

A diagonal model of image formation can be defended using a variety of arguments, including the idea that very narrowband sensors imply a diagonal transform for flat patches [15] or the argument that a surface spectral reflection can be fairly well approximated as a dimension-3 expansion in a finite-dimensional-model (FDM) basis set [16, 17]. Healey et al. use the latter assumption in [14] to support planar-patch surface normal segmentation by switching lighting environments. Another supporting argument for a diagonal model relates to the Factor Model of image formation, discussed below. The Factor Model argument has been used to justify a diagonal model for tasks such as image indexing [15] or video segmentation [18]. In this paper we are not changing the illumination.

\subsection{Factor Model of Image Formation}

In computer graphics a simple approach to approximating color for reflected light consists of multiplying the RGB triple for the illuminant times that for the surface. In terms of eq. (1) this amounts to approximating the color eq. (2) with components $b_{k}^{i}$ by

$$
b_{k}^{i} \simeq s_{k} \epsilon_{k}^{i} / \sigma_{k}, \quad k=1 . .3
$$

where $s_{k}$ is the surface color under equi-energy white light

$$
s_{k}=\int S(\lambda) q_{k}(\lambda) d \lambda
$$

and $\epsilon_{k}^{i}$ is the color of the $i$ th illuminant,

$$
\epsilon_{k}^{i}=\int I^{i}(\lambda) q_{k}(\lambda) d \lambda
$$

The camera scaling term is

$$
\sigma_{k}=\int q_{k}(\lambda) d \lambda
$$

Borges [19] carefully considered this approximation and showed that it is accurate provided the illuminant is "white enough". In practice, the light can be relatively non-white and still give accurate enough results under 
a Factor Model. (For a flat colored surface the Factor Model leads to a diagonal model of illuminant change [15]).

Here we shall make use of this approximation to derive the locus in color space for each of many uniformlycolored patches, for a curved surface.

\section{Color Ellipsoids under Piecewise-Uniform Factor Model}

\subsection{Color Ellipsoid for One Uniformly-Colored Patch: Calibration}

For a single patch with uniform surface spectral reflectance $S(\lambda)$, colors live on an ellipsoidal surface in 3dimensional in color space [3,4] since eq. (6) implies that

$$
\begin{aligned}
& 1 \equiv \boldsymbol{n}^{T} \boldsymbol{n}=\boldsymbol{\rho}^{T} \boldsymbol{G}^{T} \boldsymbol{G} \boldsymbol{\rho}=\boldsymbol{\rho}^{T} \boldsymbol{H} \boldsymbol{\rho} \\
& \text { with } \boldsymbol{G}=\boldsymbol{F}^{-1}, \boldsymbol{H}=\boldsymbol{G}^{T} \boldsymbol{G}
\end{aligned}
$$

Matrix $F$ is invertible if it is rank 3; it will be full rank if the factor matrices $B$ and $A$ are full rank, rank 3 . The latter pair of matrices are full rank if lighting directions are linearly independent and if colors $\boldsymbol{b}^{i}$ are also linearly independent. Colors $\boldsymbol{b}^{i}$ are all positive numbers and we can ensure that $\boldsymbol{A}$ is positive-definite, making $F$ also positive-definite. Thus colors lie on an ellipsoid in color space.

Note that if we employ a white calibration sphere $[13,20]$ it is quite simple to calibrate for matrix $F-$ the $k$ th row in matrix $F$ is derived from the pixel with the maximum intensity in the $k$ th color band of the sphere image.

\subsection{Ellipsoids for Many Uniformly-Colored Patches}

Suppose that we first calibrate our lighting environment using a white sphere, so that matrix $F$ in eq. (5) is known. Now suppose an unknown shape, that has a surface painted with uniform patches of color, is immersed in the calibrated lighting environment.

Let us use a concrete synthetic exemplar image for which ground truth for both colors and surface normals are known, so as to be able to compare results with known values. Fig. 1(a) shows a laser range image for a plaster bust and Fig. 1(b) shows the image resulting from a Lambertian assumption, with surface reflectance equal to the first 15 patches from the Macbeth ColorChecker standard target image [21], plus the first neutral from that target. Here we have illuminated the depth map using the three illuminants shown in Fig. 2(a), from (tilt, slant) directions $\left(-60^{\circ}, 30^{\circ}\right),\left(-120^{\circ}, 30^{\circ}\right)$, and $\left(90^{\circ}, 30^{\circ}\right)$. Camera sensitivities were taken to be as shown in Fig. 2(b). A white sphere, immersed in the same complex lighting environment, results in the image in Fig. 1(c).

These figures are printed in black and white but may be viewed in color at [22].

To indicate that eq. (5) refers to the calibration conditions, denote the original $\boldsymbol{F}$ as $\boldsymbol{F}_{\mathbf{0}}$ and its inverse as $\boldsymbol{G}_{\mathbf{0}}$. The absolute numbers in $\boldsymbol{F}_{\mathbf{0}}$ will depend on exposure control and the brightness of the illuminants. Note that we are using linear RGB's and the present method requires including linearization from any camera gamma setting as well as dark current subtraction.

Writing matrix $\boldsymbol{F}_{\mathbf{0}}$ in eq. (6) in terms of three 3 -vectors $\boldsymbol{f}_{k}, k=1 . .3$ as

$$
\boldsymbol{F}_{\mathbf{0}}=\left[\begin{array}{l}
\left(\boldsymbol{f}_{1}\right)^{T} \\
\left(\boldsymbol{f}_{2}\right)^{T} \\
\left(f_{3}\right)^{T}
\end{array}\right]
$$


we note that however we set up the lighting environment, the maximum pixel value in any channel is governed by the norm of the 3 -vector $f$ in that channel:

$$
\max (\text { pixel value })=\left\|\boldsymbol{f}_{k}\right\|, \quad k=1 . .3 \quad .
$$

To see this, denote normalized vectors by $\widehat{f}_{k}$ and note that the brightness in the $k$ th channel is

$$
\rho_{k}=\left(\boldsymbol{f}_{k}\right) \boldsymbol{n}=\left\|\boldsymbol{f}_{k}\right\|\left(\widehat{\boldsymbol{f}}_{k}\right)^{T} \boldsymbol{n} \leq\left\|\boldsymbol{f}_{k}\right\|
$$

This observation allows us to screen out possibly unphysical colors, below. Fig. 1(c) includes an indication of the direction of vectors $f_{k}$.

Suppose we now make use of a factor model of color image formation. Then in each uniformly-colored patch, since we multiply matrix $B$ by a $3 \times 3$ diagonal matrix, we also change the effective lighting environment $F$ by a diagonal transform $D$ :

$$
\boldsymbol{F}=\boldsymbol{D} \boldsymbol{F}_{\mathbf{0}} \quad, \quad \boldsymbol{D}=\operatorname{diag}\left(d_{k}\right) \equiv \operatorname{diag}\left(s_{k} / \sigma_{k}\right) \quad,
$$

with diagonal elements $d_{k}$ equal to the surface color components $s_{k}$ from eq. (8), divided by the camera scales (10), and pixel RGB is given by

$$
\rho=F n=D F_{0} n
$$

with

$$
\left(\boldsymbol{F}_{\mathbf{0}}\right)_{k j}=\sum_{i} \epsilon_{k}^{i} a_{j}^{i}
$$

Then in that patch colors are governed by a quadratic form with symmetric, positive-definite matrix $\boldsymbol{H}$ given by

$$
\boldsymbol{H}=\boldsymbol{G}^{T} \boldsymbol{G}=\boldsymbol{D}^{-1} \boldsymbol{G}_{\mathbf{0}}{ }^{T} \boldsymbol{G}_{\mathbf{0}} \boldsymbol{D}^{-1}=\boldsymbol{E} \boldsymbol{H}_{\mathbf{0}} \boldsymbol{E} \quad \text { where } \quad \boldsymbol{E} \equiv \boldsymbol{D}^{-1}, \quad \boldsymbol{H}_{\mathbf{0}}=\boldsymbol{G}_{\mathbf{0}}{ }^{T} \boldsymbol{G}_{\mathbf{0}} .
$$

Thus for pixels that derive from the same patch and that see all lights, we have the fundamental equation

$$
\boldsymbol{\rho}^{T} \boldsymbol{E} \boldsymbol{H}_{0} \boldsymbol{E} \boldsymbol{\rho}=1
$$

Surface color information is thus contained in diagonal matrix $E$; here we aim to recover this color information and also surface normals, with matrix $\boldsymbol{H}_{\mathbf{0}}$ assumed as given.

Note that to truly have an ellipsoidal surface and not a degenerate one [5] both lighting (matrix $\boldsymbol{F}_{\mathbf{0}}$ ) and surface (the collection of normals $\boldsymbol{n}$ in the patch) must be rank-3. I.e., in this paper we are assuming that surfaces are curved: the proposed method will not work for flat, planar patches.

Petrov and Antonova [10] proceed to recover an unknown matrix $\boldsymbol{H}=\boldsymbol{G}^{T} \boldsymbol{G}$ (with no factor model) using entirely local operations, by taking derivatives of eq. (11). This approach stands in contradistinction to previous methods that recover unknown matrix $\boldsymbol{H}$ for a collection of lights by using the statistics of many pixels in a patch, and so are nonlocal $[3,4,6]$. For flat uniformly-colored patches, derivatives would be zero and hence the method could not apply. Let us apply this approach to a model that assumes the factor approximation.

Taking first partial derivatives of the version of eq. (11) that separates color information from lighting information, eq. (19), and assuming matrix $E$ is constant in a patch, we have

$$
\begin{aligned}
& \boldsymbol{\rho}^{T} \boldsymbol{E} \boldsymbol{H}_{0} \boldsymbol{E} \boldsymbol{\rho}_{x}=0 \\
& \boldsymbol{\rho}^{T} \boldsymbol{E} \boldsymbol{H}_{0} \boldsymbol{E} \boldsymbol{\rho}_{y}=0
\end{aligned}
$$


Finally, taking second partial derivatives we also have

$$
\begin{aligned}
& \boldsymbol{\rho}_{x}^{T} \boldsymbol{E} \boldsymbol{H}_{\mathbf{0}} \boldsymbol{E} \boldsymbol{\rho}_{x}+\boldsymbol{\rho}^{T} \boldsymbol{E} \boldsymbol{H}_{\mathbf{0}} \boldsymbol{E} \boldsymbol{\rho}_{x x}=0 \\
& \boldsymbol{\rho}_{y}^{T} \boldsymbol{E} \boldsymbol{H}_{\mathbf{0}} \boldsymbol{E} \boldsymbol{\rho}_{x}+\boldsymbol{\rho}^{T} \boldsymbol{E} \boldsymbol{H}_{\mathbf{0}} \boldsymbol{E} \boldsymbol{\rho}_{x y}=0 \\
& \boldsymbol{\rho}_{y}^{T} \boldsymbol{E} \boldsymbol{H}_{\mathbf{0}} \boldsymbol{E} \boldsymbol{\rho}_{y}+\boldsymbol{\rho}^{T} \boldsymbol{E} \boldsymbol{H}_{\mathbf{0}} \boldsymbol{E} \boldsymbol{\rho}_{y y}=0
\end{aligned}
$$

However, Petrov and Antonova [10] indicate that a solution of the above set of six equations eqs. (19, 20, 21 ) is fragile: "For reasons that are not clear, the algorithm sometimes fails to recover a unique rotation." "... and we have to use a careful strategy for resolving the system." "It is necessary that a computer implementation of [their] formulae 12 to use carefully regularized algorithms of matrix inversion and derivative estimation." Here we wish to derive a solution for a situation that is simpler in principle, albeit with known lighting environment, in order to be able to dispense with the last three equations, eqs. (21). Since the method uses lower order partial derivatives in the image, one expects much greater stability than for Petrov et al.'s algorithm. In a knownlighting, color photometric stereo setting, we should be able to solve for the three unknowns $e_{k}, k=1 . .3$ in the diagonal matrix

$$
\boldsymbol{E} \equiv \operatorname{diag}\left(e_{1}, e_{2}, e_{3}\right)=\operatorname{diag}\left(1 / d_{1}, 1 / d_{2}, 1 / d_{3}\right)=\operatorname{diag}\left(\sigma_{k} / s_{k}\right)
$$

by making use of the three equations $(19,20)$ only. Since each solution is local, we thus find color locally in each uniformly-colored patch, with problems only on a color edge or at a pixel that does not see all lights.

\section{Solution of Color-Ellipsoid Equations}

We can begin to solve the set of equations $(19,20)$ by first noting that eqs. (20) can be written

$$
\rho^{T} \boldsymbol{E} H_{0} E \nabla \rho=0
$$

Here, the gradient is a spatial operator applied to each color component separately. Although it is not standard to apply a gradient to a vector in this way, there is no ambiguity here because the vector components span a space different from the space in which the vector operator is applied. Hence the color-vector components act as separate scalars with respect to spatial operations. Color as a vector-valued function is not like electric field (in which each component is associated with a spatial direction).

Thus a solution is necessarily of the form (cf. the similar solution in [10])

$$
\boldsymbol{E} \boldsymbol{H}_{\mathbf{0}} \boldsymbol{E} \boldsymbol{\rho}=\frac{\boldsymbol{\rho}_{x} \times \boldsymbol{\rho}_{y}}{\boldsymbol{\rho}^{T}\left(\boldsymbol{\rho}_{x} \times \boldsymbol{\rho}_{y}\right)} \equiv \boldsymbol{v} .
$$

This vector is correctly orthogonal to $\nabla \rho$ and also satisfies eq.(19).

At this point, since we have separated color from lighting and as well we mean to provide a known matrix $\boldsymbol{H}_{\mathbf{0}}$, we part company with Petrov and Antonova's approach [10].

Now our set of nonlinear equations to solve for the three unknowns $e_{k}$ is the set of three equations (24). The right hand side of eq. (24) consists of a vector that is calculated from measured data only.

With the definition (24) of vector $v$, eq. (19) is automatically satisfied and the components of vector $v$ must obey the condition

$$
\boldsymbol{\rho}^{T} \boldsymbol{v}=1 \quad .
$$

Note that if we happened to know the normal $n$ at the current pixel, then in fact substitution of (16) into the left hand side of eq. (24) would allow us to explicitly write vector $\boldsymbol{v}$ as $\boldsymbol{v}=\boldsymbol{E} \boldsymbol{G}_{\mathbf{0}}{ }^{T} \boldsymbol{n}$. 
Eq.(24) constitutes three nonlinear equations in the unknown color-ratio triple $\boldsymbol{e}$. The solution is given in Appendix A; its heart is the set of roots of a quartic equation in the square of one of the components of $e$. Since $e$ is necessarily positive because it consists of the inverse of a set of colors, there are at most four real solutions. Below, we explore the meaning of these solutions - they amount to solutions of all points on a given ellipsoid where the ellipsoid normal equals a given vector, divided by the components of the ellipsoid point itself. The fact that there may be more than one root can result in an ambiguity. Fortunately, this ambiguity can usually be resolved by the fact that only one solution leads to a maximum RGB value in the physically-possible range.

Firstly, we note that the factor model of color may lead to equations with no real solutions - the actual image $\rho$ may be too far from a factor model approximation. And in fact below, in $\S 6$, we find that there are some pixels that give no solution. Also, there may be pixels that lead to multiple solutions.

Nevertheless in most situations if there are multiple solutions at least some of them will lead to negative values of some color components $\boldsymbol{e}$. The reason is that, while the solution is a quartic equation in one of the components of $\boldsymbol{e}$, say $\left(e_{3}\right)^{2}$, so that we usually find some positive solutions for $e_{3}$, the remaining equations for $e_{1}$ and $e_{2}$ may yield negative values. We exclude any solution that does so.

As well, some solutions may be unphysical. Recall from eq. (13) that the maximum pixel value in any channel is given by the norm of the corresponding row of matrix $\boldsymbol{F}_{\mathbf{0}}$. However, from eq. (15) we also have that if the surface is not white, the maximum pixel value is multiplied by the diagonal component $d_{k}$ for that channel. Thus values recovered for elements $d_{k}$ should be less than unity or we could have colors that are whiter than white and thus impossible. Therefore a useful criterion for culling incorrect solutions $e_{k}=1 / d_{k}$ is that these should obey the constraint

$$
e_{k} \geq 1 \quad \text {. }
$$

Finally, in any remaining case for which that there are still multiple solutions, the correct one may be disambiguated by a further derivative of eq. (24):

$$
E H E \nabla \rho=\nabla v
$$

For surviving multiple solutions, we take that $e$ with minimum value of

$$
\left\|\boldsymbol{E} \boldsymbol{H} \boldsymbol{E} \boldsymbol{\rho}_{x}-\boldsymbol{v}_{x}\right\|^{2}+\left\|\boldsymbol{E} \boldsymbol{H} \boldsymbol{E} \boldsymbol{\rho}_{y}-\boldsymbol{v}_{y}\right\|^{2}
$$

Note that this is not the same as using the set of higher-order derivative equations (21) to find the color, since here we may merely have to select the correct solution, rather than using higher order derivatives to solve for a solution. For completeness, Petrov and Antonova's solution for matrix $\boldsymbol{H}$ is included in Appendix B.

The color image Fig. 1(b) can clearly be easily segmented by a variety of means [23]. If just some pixels are available in each segment, nevertheless we may still recover approximate surface normals, not just where pixels have solutions for color-ratios $e$, but throughout the patch. Here we apply a robust LMS mode-finder [24] to the problem of distinguishing the statistically best value of each component of $e$ in a patch. Then, applying that same diagonal matrix $E$ throughout the patch, the approximation of surface normals is

$$
n \simeq G_{0} E \rho \quad \text { with } G_{0}=F_{0}^{-1} .
$$

Since we are partly relying on an image segmentation we might ask why don't we simply estimate matrix $\boldsymbol{H}$ in each patch separately and thus recover surface normals? The answer is that simply estimating $\boldsymbol{H}$, or better yet $\boldsymbol{F}$ using some assumptions (cf. [9]), does no better at producing an estimate of color for us, and may in fact do worse. For suppose we first estimate matrix $\boldsymbol{H}=\boldsymbol{G}^{T} \boldsymbol{G}$ from RGB values $\boldsymbol{\rho}$ in a patch, e.g. employing a Least Median of Squares estimate, as advocated in [6] or using Petrov and Antonova's solution 
method. Firstly, unless $\boldsymbol{H}$ is exactly diagonally related to $\boldsymbol{H}_{0}$, there is no solution for $\boldsymbol{E}$ from $\boldsymbol{E} \boldsymbol{H}_{0} \boldsymbol{E}=$ $\boldsymbol{H}$. Secondly, suppose instead that we find a least squares estimate of inverse ratio $\boldsymbol{E}=\operatorname{diag}(\boldsymbol{e})$ via a minimization $\min \sum\left\|\boldsymbol{E} \boldsymbol{H}_{0} \boldsymbol{E}-\boldsymbol{H}\right\|^{2}$. Then $e_{1}=e_{2}=e_{3}=0$ is now a solution found (the constant term drops out after differentiation of the least squares objective); this solution is not present in the method outlined in this paper. Moreover, since we have introduced error with our statistical or other estimate of $\boldsymbol{H}$, not all the solutions found in the present approach may indeed be found by a minimization and, worse, more solutions are introduced.

\section{Spectral Sharpening}

The accuracy of the method presented depends upon that of the factor model eq. (7), and the accuracy of the latter can often be improved by carrying out a spectral sharpening operation on the camera sensors of Fig. 2(b). Spectral sharpening [12] consists of a $3 \times 3$ matrix transform $M$ on the camera sensor set $\boldsymbol{Q}$, such that each curve's "energy" is better concentrated in a small sharpening interval, a subset of the visible wavelength range. The rationale here is that for perfectly sharp, delta-function sensors the factor model approximation (7) holds exactly.

Fig. 2(c) shows the camera sensors sharpened in wavelength intervals [600-640], [520-560], and [450-490] in the $\mathrm{R}, \mathrm{G}$, and $\mathrm{B}$ channels respectively.

Note that the derived sensors are not all-positive. Nevertheless, the sensors shown in fact produce only positive RGB values (cf. [25]), and yet are very close to the best possible sharpened sensors with negative lobes allowed (that do produce negative colors). The utility of using sharpened sensors that produce only non-negative RGB values is that the condition (26) still holds and can be used to constrain correct solutions.

In the color-transformed space, all the statements made so far still apply; in particular, we can still use eq. (29) to derive recovered surface normals, provided that all quantities are written in the sharpened color space.

If matrix $M$ produces sharpened sensors $Q^{\prime}$ from unsharpened ones $Q$ via

$$
Q^{\prime}=M Q
$$

then colors in the transformed space are given by

$$
\rho^{\prime}=M \rho
$$

Hence all other color quantities are also transformed in the same way:

$$
\begin{aligned}
& \boldsymbol{b}^{\prime}=M \boldsymbol{b}, \\
& \boldsymbol{\sigma}^{\prime}=M \boldsymbol{\sigma}, \\
& \boldsymbol{s}^{\prime}=M \boldsymbol{s}, \\
& \boldsymbol{B}^{\prime}=M B \\
& \boldsymbol{F}_{\mathbf{0}}^{\prime}=M \boldsymbol{F}_{\mathbf{0}} \\
& \boldsymbol{F}^{\prime}=M \boldsymbol{F}
\end{aligned}
$$

where $s=\operatorname{diag}\left(s_{1}, s_{2}, s_{3}\right)$.

Thus the algorithm we employ is as follows:

1. Form the image in spectrally-sharpened color space, by matrix multiplication of the original image:

$$
\rho^{\prime}=M \rho
$$


2. Form image derivatives $\rho_{x, y}^{\prime}$ and vectors $f^{\prime}$.

3. Derive a solution set of factor model multipliers $\boldsymbol{E}^{\prime}=\operatorname{diag}\left(e_{k}^{\prime}\right)$ and surface normal vectors $\boldsymbol{n}$

$$
n \simeq G_{0}{ }^{\prime} E^{\prime} \rho^{\prime}
$$

4. The inverse of our solution $E^{\prime}$ gives a color-multiplier diagonal matrix

$$
D^{\prime}=\operatorname{diag}\left(d_{k}^{\prime}\right) \equiv \operatorname{diag}\left(s_{k}^{\prime} / \sigma_{k}^{\prime}\right)
$$

so that colors $s_{k}^{\prime}$ defined in the transformed space are given by

$$
\operatorname{diag}\left(s_{k}^{\prime}\right)=D^{\prime} \operatorname{diag}\left(\sigma_{k}^{\prime}\right) \quad \text {. }
$$

5. Finally, transform the uniform patch colors back to the original color space via

$$
\operatorname{diag}\left(s_{k}\right)=M^{-1} \operatorname{diag}\left(s_{k}^{\prime}\right)
$$

Colors $s_{k}$ are the colors of the surface as seen under white light.

In trials, we found that spectral sharpening improved the performance of the algorithm, for the camera data used here.

\section{Simulation Results}

Firstly, it is of interest just how accurate the factor model of color is. For the 16 patches in Fig. 1(b), let us consider how well colors $\boldsymbol{b}^{i}$, under light $I^{i}(\lambda)$, are approximated. The correct colors $\boldsymbol{b}$ are given by eq. (2), whereas the factor model approximations $\hat{b}$ are given by eq. (7). For each of the 16 patches and each of the three illuminants used, let us use an error indicator

$$
\text { error } \equiv\|\boldsymbol{b}-\widehat{b}\| /\|\boldsymbol{b}\| \quad,
$$

written as a percentage. Here we find that the median error value for colors $b$ is $8.2 \%$. However, the maximum error is $54.5 \%$, so that for at least some of the patches and illuminants, the factor model breaks down.

However, spectral sharpening substantially improves matters for the camera sensor data used here. Fig. 2(c) shows the spectrally sharpened camera sensors. Although these curves have negative lobes, nevertheless they produce only non-negative RGB values.

For sharpened colors, the median factor model error is now 5.4\% and the maximum error is $44.9 \%$.

Let us determine which patches give the worst errors. Patches are numbered from 1 to 16 in Fig. 1(b) in row-major order from top-left to bottom-right. Averaging over all lights, the minimum error of $1.3 \%$ results from patch \#16, the neutral color. The maximum error of $29.4 \%$ is for patch \#15, which is a strong red. The largest errors are thus for the most saturated colors: other high errors are $13.2 \%$ for \#7, a strong orange, $12.2 \%$ for \#9, a moderate red, and $18.5 \%$ for \#10, a deep purple. Thus we could expect problems from these patches. However, another main source of error is shadowing, since the color formation model breaks down in shadows. As we shall see below, problems also turn out to occur in patches \#4, 9, and 12. Patches \#4 and 12 are recovered inaccurately simply because there is a dearth of data in those patches: the figure intersects with the checkerboard of color mapped onto the range image in very few pixels. Patch \#9 has problems because it is most in shadow 
from the lights: $83 \%$ of the patch lies in shadow from at least one of the lights. Recall that the algorithm flags problems if no solution is possible, or if negative or impossible colors arise. For the problematic patches identified, not many pixels are available for solution.

Running the algorithm presented in $\S 4$ and 5 and in Appendix A, we arrive at estimates for colors $s$ in each patch. Table 1 gives the number of pixels that are possibly available to the algorithm to use in determining $e$ in a patch, for a $128 \times 128$ image. As well, Table 1 shows how many pixels are actually used. For several of the patches, very few pixels are available for a solution. Fig. 3(a) shows areas of the image where in fact the surface is in shadow from at least one of the lights. White pixels are in shadow. This mask image is not recovered from the algorithm but is shown simply for comparison with the mask image of Fig. 3(b); here, white pixels show where at least one solution exists. We note that solutions do not exist where shadows lie. As well, there are problems in some patches where the factor model does not do well.

Nevertheless we may still find the ratios $e^{\prime}$ in each patch using a robust mode-finder separately for each component $\epsilon_{k}^{\prime}$ for each patch, and from them derive unsharpened colors $s$. Here, we use the LMS method, for one-dimensional data [24]. Table 1 shows the errors, defined after the fashion of eq. (37), for vectors $s$. The median error is $7.1 \%$, which is remarkably good considering how few pixels were used in some patches. If we are mostly interested in hue and saturation, rather than 3-dimensional color, then we can examine the errors in chromaticity, defined as the 2-vector $(r, g)=(R, G) /(R+G+B)$. Table 1 shows the errors for chromaticity; the median error value is $3.3 \%$.

Using the robustly-determined inverse ratios $e^{\prime}$ we may apply eq. (33) for surface normal recovery either indiscriminately to every pixel in a patch, or else just to selected pixels. We may wish to apply that equation only to the pixels with solutions for $e^{\prime}$, shown in Fig. 3(b); or else make use of the inlier/outlier discrimination estimate provided automatically by the LMS mode estimator, and apply eq. (33) just to inliers. Here we apply the normal recovery equation to all pixels, with result as shown in Fig. 4(a). This figure shows the recovered surface normals, illuminated from direction $(0,0,1)$.

Note that eq. (33) does not, in fact, guarantee normalized, length-1, normal vectors $\boldsymbol{n}$; in fact, most normal vectors recovered are indeed length-1, with exceptions principally at color borders and at the occluding boundary.

In comparison, Fig. 4(b) shows the actual surface normals, shaded in the same way. These figures show that the recovery method has performed quite well. A more explicit measure of goodness is provided by an angular error measure $\cos ^{-1}(\hat{\boldsymbol{n}} \cdot \boldsymbol{n} /(\|\hat{\boldsymbol{n}}\|\|\boldsymbol{n}\|))$, applied to normal vector estimates $\hat{\boldsymbol{n}}$. Recovered surface normals have a median angular error of only $4.96^{\circ}$.

Fig. 5(a) shows the recovered surface with recovered color as seen under white light. For comparison, Fig. 5(b) shows the actual surface and actual colors, as seen under white light. The worst color error, as shown in Table 1, is in patch \#15, the patch that has the most saturated color. Considering the small number of pixels available in patches \#4, 9 , and 12 , the chromaticity errors are surprisingly small, comparable to those of the other patches.

\section{Meaning of Color Equations}

We can better understand why there may be multiple solutions to eq. (24) by defining a new diagonal matrix composed from the elements of the RGB vector $\rho$ :

$$
\boldsymbol{R}=\operatorname{diag}\left(\rho_{1}, \rho_{2}, \rho_{3}\right)
$$


Then commuting $E$ and $\boldsymbol{R}$, the condition eq. (19) may be rewritten

$$
\boldsymbol{e}^{T}\left(\boldsymbol{R} \boldsymbol{H}_{0} \boldsymbol{R}\right) \boldsymbol{e}=1
$$

Multiplying the main equation which must be solved, eq. (24), by $R$ yields

$$
E\left(R H_{0} R\right) e=R v
$$

Thus at any pixel $\boldsymbol{\rho}$ (with matrix $\boldsymbol{R}$ ), according to eq. (39) we seek a solution vector $\boldsymbol{e}$ that lies on the quadratic form $\left(\boldsymbol{R} \boldsymbol{H}_{0} \boldsymbol{R}\right)$. At a solution color-ratio vector $\boldsymbol{e}$, we must also have the normal vector to the ellipsoid ( $R H_{0} R$ ) equal to the given vector $R v$, multiplied by the diagonal matrix $E^{-1}=D$ :

$$
\left(R H_{0} R\right) e=D(R v) .
$$

(The left-hand-side of the above equation is the normal vector to the quadratic form at point $e$ because for a quadratic form $\boldsymbol{A}$, taking the derivative of $\boldsymbol{x}^{T} \boldsymbol{A} \boldsymbol{x}$ gives $2 \boldsymbol{A} \boldsymbol{x}$, so that the normal vector at $\boldsymbol{x}$ is proportional to $\boldsymbol{A} \boldsymbol{x}$. Only along the principal axes is the normal along $\boldsymbol{x}$.)

Eq. (41) states that at a solution point $e$ on the quadratic form $\left(\boldsymbol{R} \boldsymbol{H}_{0} \boldsymbol{R}\right)$, the normal vector equals a specified vector $(\boldsymbol{R} \boldsymbol{v})$ divided by the components of the point $\boldsymbol{e}=\left(e_{1}, e_{2}, e_{3}\right)^{T}$.

Fig. 6 shows the quadratic form for a particular RGB point $\rho$ for the Mozart image of $\S 6$, in the $e_{1}, e_{2}, e_{3}$ space. For this particular color point, chosen because eqs. $(19,24)$ have four solutions there, two solutions have negative values of $e_{3}$, but there are two solutions properly in the first octant. The figure shows the vector $\left(\boldsymbol{R} H_{0} R\right) e$ for each of the four solution points $e$. None of these vectors equals the normal vector at its associated solution point $e$, but each vector equals the same vector $\boldsymbol{R} \boldsymbol{v}$, divided by the components of $\boldsymbol{e}$.

\section{Conclusions}

We have shown that, by assuming known light sources and a factor model for illuminant-surface interaction, it is possible to recover surface normals using only half the equations posed by Petrov, and that it is possible to recover color as well. Color solutions available in each patch are extended throughout that patch, yielding approximate surface normal recovery at all pixels, and results are quite encouraging both for recovered normals and colors.

In $\S 4$ we noted that simply solving for the color space ellipsoid in a patch does not necessarily tell us what the color is there. This is because a minimization of $\sum\left\|\boldsymbol{E} \boldsymbol{H}_{\mathbf{0}} \boldsymbol{E}-\boldsymbol{H}\right\|^{2}$ usually produces more, not fewer solutions $e$ and may miss the correct one produced by the present method.

Another possibility is a minimization at each pixel that views eq. (24) relating vector $\boldsymbol{e}$ to vector $v$ as a statistical statement: suppose we perform a minimization $\min \|\boldsymbol{E} \boldsymbol{H} \boldsymbol{E} \boldsymbol{\rho}-\boldsymbol{v}\|^{2}$. This indeed finds all the solutions that eqs. $(19,24)$ do, along with a new incorrect solution $e_{1}=e_{2}=e_{3}=0$, but as well there is also a collection of new, spurious local minima.

Another approach might be a color approximation with higher order nonlinear terms than the factor model. However, such an approach would detract from the pleasing simplicity of that model.

Perhaps most important would be an investigation into just what illuminant colors and positions could most reliably give good results in the method presented here. The matrices involved are most likely to be full rank if lighting colors are saturated (i.e., colorful); whereas the factor model works best for desaturated lights. The color ellipsoids are more sphere-like if the lights are well separated in space; however that circumstance exactly produces the most self-shadowing. Thus there are number of tradeoffs that must be reconciled. That task will be pursued elsewhere. 


\section{Appendix A: Solution of Color Equations}

Firstly the constraint (25), which amounts to eq. (19), implies

$$
v_{3}=\left(1-\rho_{1} v_{1}-\rho_{2} v_{2}\right) / \rho_{3}
$$

so that only two components of $v$ need be computed.

We need to solve the set of equations (24). Simplifying notation by writing $\left(\boldsymbol{H}_{\mathbf{0}}\right)_{j k}$ as $H_{j k}$, eq. (24) reads

$$
\begin{aligned}
& e_{1}^{2} H_{11} \rho_{1}+e_{1} e_{2} H_{12} \rho_{2}+e_{1} e_{3} H_{13} \rho_{3}=v_{1} \\
& e_{1} e_{2} H_{12} \rho_{1}+e_{2}^{2} H_{22} \rho_{2}+e_{2} e_{3} H_{23} \rho_{3}=v_{2} \\
& e_{1} e_{3} H_{13} \rho_{1}+e_{2} e_{3} H_{23} \rho_{2}+e_{3}^{2} H_{33} \rho_{3}=v_{3}
\end{aligned}
$$

or, if we make the substitutions $x=e_{1}, y=e_{2}, z=e_{3}$, and $\alpha_{k}=H_{1 k} \rho_{k} / v_{1}$, $\beta_{k}=H_{2 k} \rho_{k} / v_{2}, \gamma_{k}=H_{3 k} \rho_{k} / v_{3}$, these equations read

$$
\begin{aligned}
& \alpha_{1} x+\alpha_{2} y+\alpha_{3} z=1 / x \\
& \beta_{1} x+\beta_{2} y+\beta_{3} z=1 / y \\
& \gamma_{1} x+\gamma_{2} y+\gamma_{3} z=1 / z
\end{aligned}
$$

Defining, further, $p=x / z, q=y / z$, then the ratios of eqs. (A3) and (A5), and eqs. (A4) and (A5), give the two equations

$$
\begin{aligned}
& \gamma_{1} p+\gamma_{2} q+\gamma_{3}=\alpha_{1} p^{2}+\alpha_{2} p q+\alpha_{3} p \\
& \gamma_{1} p+\gamma_{2} q+\gamma_{3}=\beta_{1} p q+\beta_{2} q^{2}+\beta_{3} q
\end{aligned}
$$

We can solve (A6) for $q$ easily, since the equation is linear in $q$. Substituting into (A7) yields a quartic equation in $p$, as follows:

$$
\phi(p)=d_{0}+d_{1} p+d_{2} p^{2}+d_{3} p^{3}+d_{4} p^{4}=0
$$

with coefficients

$$
\begin{aligned}
d_{0}= & -\beta_{2}\left(\gamma_{3}\right)^{2}+\beta_{3} \gamma_{2} \gamma_{3} \\
d_{1}= & -\alpha_{2} \beta_{3} \gamma_{3}-\alpha_{2} \gamma_{2} \gamma_{3}+2 \alpha_{3} \beta_{2} \gamma_{3}-\alpha_{3} \beta_{3} \gamma_{2}+\alpha_{3}\left(\gamma_{2}\right)^{2}+ \\
& \beta_{1} \gamma_{2} \gamma_{3}-2 \beta_{2} \gamma_{1} \gamma_{3}+\beta_{3} \gamma_{1} \gamma_{2} \\
d_{2}= & 2 \alpha_{1} \beta_{2} \gamma_{3}-\alpha_{1} \beta_{3} \gamma_{2}+\alpha_{1}\left(\gamma_{2}\right)^{2}+\left(\alpha_{2}\right)^{2} \gamma_{3}+\alpha_{2} \alpha_{3} \beta_{3}- \\
& \alpha_{2} \alpha_{3} \gamma_{2}-\alpha_{2} \beta_{1} \gamma_{3}-\alpha_{2} \beta_{3} \gamma_{1}-\alpha_{2} \gamma_{1} \gamma_{2}-\left(\alpha_{3}\right)^{2} \\
& \beta_{2}-\alpha_{3} \beta_{1} \gamma_{2}+2 \alpha_{3} \beta_{2} \gamma_{1}+\beta_{1} \gamma_{1} \gamma_{2}-\beta_{2}\left(\gamma_{1}\right)^{2} \\
d_{3}= & \alpha_{1} \alpha_{2} \beta_{3}-\alpha_{1} \alpha_{2} \gamma_{2}-2 \alpha_{1} \alpha_{3} \beta_{2}-\alpha_{1} \beta_{1} \gamma_{2}+2 \alpha_{1} \beta_{2} \gamma_{1}+ \\
& \left(\alpha_{2}\right)^{2} \gamma_{1}+\alpha_{2} \alpha_{3} \beta_{1}-\alpha_{2} \beta_{1} \gamma_{1} \\
d_{4}= & -\left(\alpha_{1}\right)^{2} \beta_{2}+\alpha_{1} \alpha_{2} \beta_{1}
\end{aligned}
$$

Now we can substitute the solution for $p$ back into (A6) and find $q$. To find $z$, we divide eq. (A5) by $z$, yielding an equation for $z^{2}$ in $p$ and $q$. We take the positive square root solution for $z$, and then retrieve the other components via $x=p z, y=q z$.

Thus solutions for all components of $e$ can be written in terms of the square of one of the components, say $\left(e_{3}\right)^{2}$, in terms of a quartic function $f$ with $\left(e_{3}\right)^{2}=f\left(\boldsymbol{H}_{\mathbf{0}}\right)$. 


\section{Appendix B: Recovery of Quadratic Form from Higher-Order Equations}

For completeness, we also show Petrov and Antonova's solution for eqs. (19), (20), and (21) for recovering the quadratic form matrix $\boldsymbol{H}$. (They do not recover color; recovery of surface normals requires subsequent simulated annealing in each patch.)

Since they do not use a factor model, read $\boldsymbol{H}$ for $\boldsymbol{E} \boldsymbol{H}_{0} \boldsymbol{E}$ in eq. (24). Their solution for matrix $\boldsymbol{H}$ is given by inverting the set of equations (20), (21) once the solution (24) is substituted. Eqs. (20,21), once (24) is inserted, are as follows:

$$
\begin{aligned}
& {\left[\begin{array}{l}
\boldsymbol{\rho}^{T} \\
\boldsymbol{\rho}_{x}{ }^{T} \\
\boldsymbol{\rho}_{y}{ }^{T}
\end{array}\right] \boldsymbol{H} \boldsymbol{\rho}_{x}=\left[\begin{array}{l}
0 \\
\boldsymbol{\rho}_{x x}{ }^{T} \boldsymbol{v} \\
\boldsymbol{\rho}_{x y}{ }^{T} \boldsymbol{v}
\end{array}\right]} \\
& {\left[\begin{array}{l}
\boldsymbol{\rho}^{T} \\
\boldsymbol{\rho}_{x}{ }^{T} \\
\boldsymbol{\rho}_{y}{ }^{T}
\end{array}\right] \boldsymbol{H} \boldsymbol{\rho}_{y}=\left[\begin{array}{l}
0 \\
\boldsymbol{\rho}_{x y}{ }^{T} \boldsymbol{v} \\
\boldsymbol{\rho}_{y y} \boldsymbol{v}
\end{array}\right]}
\end{aligned}
$$

and combining these a solution for $\boldsymbol{H}$ follows.

\section{References}

[1] L.L. Kontsevich. Shape reconstruction for uniformly colored and illuminated object from its twodimensional projection. In Data Processing in Complex Information Systems, pages 16-19, 1986. In Russian.

[2] A. P. Petrov. Color and Grassman-Cayley coordinates of shape. In B. E. Rogowitz, M. H. Brill, and J. P. Allebach, editors, Human Vision, Visual Processing and Digital Display II, volume 1453, pages 342-352. SPIE, 1991.

[3] M.S. Drew. Shape from color. Technical Report CSS/LCCR TR 92-07, Simon Fraser University School of Computing Science, 1992. Available using ftp:// fas.sfu.ca / pub / cs / techreports / 1992 / CSS-LCCR9207.ps.Z.

[4] L. L. Kontsevich, A. P. Petrov, and I. S. Vergelskaya. Reconstruction of shape from shading in color images. J. Opt. Soc. Am. A, 11:1047-1052, 1994.

[5] A. P. Petrov and L. L. Kontsevich. Properties of color images of surfaces under multiple illuminants. J. Opt. Soc. Am. A, 11:2745-2749, 1994.

[6] M.S. Drew. Robust specularity detection from a single multi-illuminant color image. CVGIP: Image Understanding, 59:320-327, 1994.

[7] M. S. Drew and L. L. Kontsevich. Closed-form attitude determination under spectrally varying illumination. In Proc. IEEE Comp. Soc. Conf. on Comp. Vis. and Patt. Rec., pages 985-990, 1994.

[8] M.S. Drew. Reduction of rank-reduced orientation-from-color problem with many unknown lights to two-image known-illuminant photometric stereo. In International Symposium on Computer Vision, Coral Gables, FL, Nov.21-23, pages 419-424. IEEE, 1995. 
[9] M.S. Drew. Direct solution of orientation-from-color problem using a modification of Pentland's light source direction estimator. Computer Vision and Image Understanding, 64:286-299, 1996.

[10] A. P. Petrov and G.N. Antonova. Resolving the color-image irradiance equation. Color Research and Application, 21:97-103, 1996.

[11] B. V. Funt, M. S. Drew, and M. Brockington. Recovering shading from color images. In G. Sandini, editor, ECCV-92: Second European Conference on Computer Vision, pages 124-132. Springer-Verlag, May 1992.

[12] G.D. Finlayson, M.S. Drew, and B.V. Funt. Spectral sharpening: sensor transformations for improved color constancy. J. Opt. Soc. Am. A, 11(5):1553-1563, May 1994.

[13] R. J. Woodham. Gradient and curvature from the photometric-stereo method, including local confidence estimation. J. Opt. Soc. Am. A, 11:3050-3068, 1994.

[14] G. Healey and L. Wang. Three-dimensional surface segmentation using multicolored illumination. Optical Eng., 37:1553-1562, 1998.

[15] M.S. Drew, J. Wei, and Z.N. Li. Illumination-invariant color object recognition via compressed chromaticity histograms of color-channel-normalized images. In ICCV98, pages 533-540. IEEE, 1998.

[16] G.D. Finlayson, M.S. Drew, and B.V. Funt. Color constancy: diagonal transforms suffice. J. Opt. Soc. Am. A, 11(11):3011-3019, Nov. 1994. Special issue on physics-based vision.

[17] G. Healey and D. Slater. Global color constancy: Recognition of objects by use of illumination invariant properties of color distributions. J. Opt. Soc. Am. A, 11:3003-3010, 1994.

[18] M. S. Drew, J. Wei, and Z.N. Li. Illumination-invariant image retrieval and video segmentation. Pattern Recognition, 32:1369-1388, 1999.

[19] C.F. Borges. Trichromatic approximation method for surface illumination. J. Opt. Soc. Am. A, 8:1319$1323,1991$.

[20] M. S. Drew. Photometric stereo without multiple images. In Human Vision and Electronic Imaging, volume 3016, pages 369-380. SPIE/IS\&T, 1997. ftp: // fas.sfu.ca / pub / cs / mark / Spie97 / spie97.html.

[21] C.S. McCamy, H. Marcus, and J.G. Davidson. A color-rendition chart. J. App. Photog. Eng., 2:95-99, 1976.

[22] Color figures may be viewed at http://www.cs.sfu.ca/people/Faculty/Drew/ftp/Josa00/.

[23] W. Skarbek and A. Koschan. Colout image segmentation - A survey, 1994. Technical University of Berlin. Technischer Bericht 94-32.

[24] P. J. Rousseeuw and A. M. Leroy. Robust Regression and Outlier Detection. Wiley, 1987.

[25] G.D. Finlayson and M.S. Drew. Positive Bradford curves through sharpening. In 7th Color Imaging Conference: Color, Science, Systems and Applications., pages 227-232. Society for Imaging Science \& Technology (IS\&T)/Society for Information Display (SID) joint conference, 1999. 


\section{Table Caption}

Table 1: For a $128 \times 128$ image, patches intersect the figure in Poss.Pixels points. The actual number of pixels used in determining color is shown as Actual Pixels. Percentage errors in recovered color and chromaticity is shown as Color Error and Chromaticity Error. The median color error is $7.1 \%$ and the median chromaticity error is $3.3 \%$.

\section{Figure Captions}

Fig. 1: (a): Radar range image. (b): Lambertian surface with surface reflectance of 16 Macbeth patches, simultaneously illuminated by three illuminants. (c): White Lambertian sphere in same lighting. Symbols $r, g, b$ mark location of maxima in $\mathrm{R}, \mathrm{G}$, and $\mathrm{B}$ channels.

Fig. 2: (a): Three illuminants. Equi-energy white light is also shown. (b): Camera sensitivities. (c): Sharpened camera sensitivities.

Fig. 3: (a): Pixels self-shadowed from at least one light. (b): Pixels with solutions for ratios $e^{\prime}$.

Fig. 4: (a): Recovered normals, shaded from $(0,0,1)$. (b): Ground truth normal vectors, also shaded from $(0,0,1)$.

Fig. 5: (a): Recovered surface with recovered color as seen under white light. (b): Ground truth surface and colors under white light.

Fig. 6: Ellipsoid in $e_{1}, e_{2}, e_{3}$ space associated with a particular RGB point, with four solutions for normal vector equal to a given vector divided by the ellipsoid coordinates. 


\begin{tabular}{|l|c|c|c|c|c|c|c|c|}
\hline Patch \# & 1 & 2 & 3 & 4 & 5 & 6 & 7 & 8 \\
\hline \hline Poss. Pixels & 231 & 847 & 828 & 75 & 602 & 1024 & 1024 & 332 \\
\hline Actual Pixels & 32 & 281 & 384 & 7 & 253 & 728 & 524 & 70 \\
\hline Color Error & $9.3 \%$ & $3.8 \%$ & $3.1 \%$ & $19.3 \%$ & $2.1 \%$ & $7.4 \%$ & $1.1 \%$ & $23.7 \%$ \\
\hline Chromaticity Error & $2.2 \%$ & $2.8 \%$ & $2.3 \%$ & $6.4 \%$ & $2.9 \%$ & $3.7 \%$ & $0.9 \%$ & $7.1 \%$ \\
\hline \hline Patch \# & 9 & 10 & 11 & 12 & 13 & 14 & 15 & 16 \\
\hline \hline Poss. Pixels & 280 & 1024 & 1013 & 59 & 611 & 1024 & 1024 & 744 \\
\hline Actual Pixels & 17 & 407 & 397 & 7 & 245 & 703 & 250 & 304 \\
\hline Color Error & $29.0 \%$ & $15.6 \%$ & $3.0 \%$ & $47.4 \%$ & $6.9 \%$ & $5.1 \%$ & $53.4 \%$ & $2.0 \%$ \\
\hline Chromaticity Error & $10.0 \%$ & $6.6 \%$ & $2.1 \%$ & $18.5 \%$ & $21.0 \%$ & $4.6 \%$ & $2.9 \%$ & $1.2 \%$ \\
\hline
\end{tabular}

Table 1: 


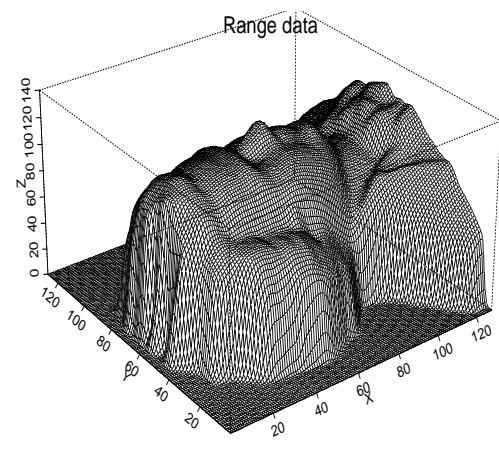

(a)

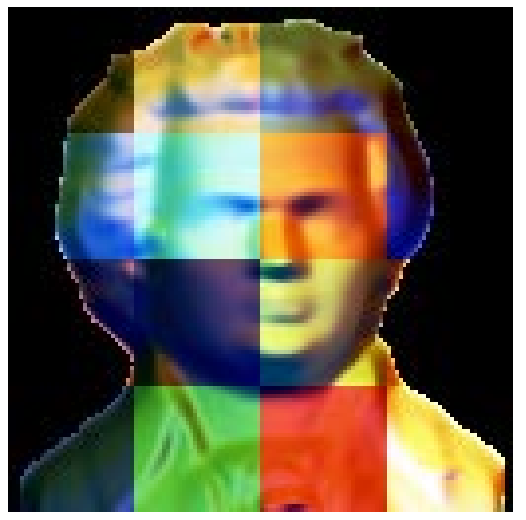

(b)

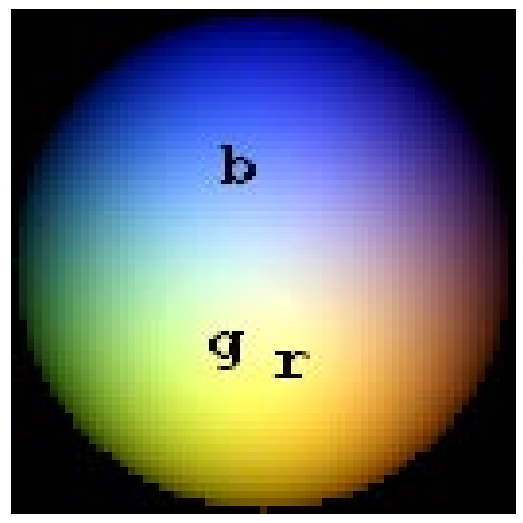

(c)

Fig. 1:

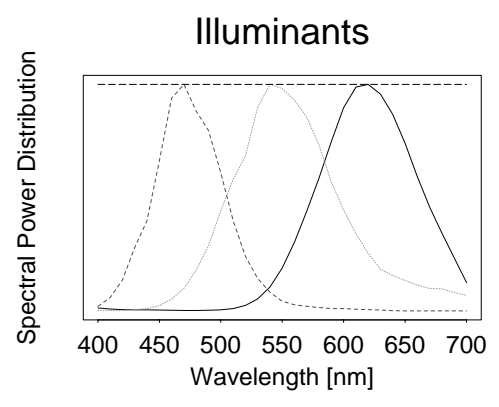

(a)

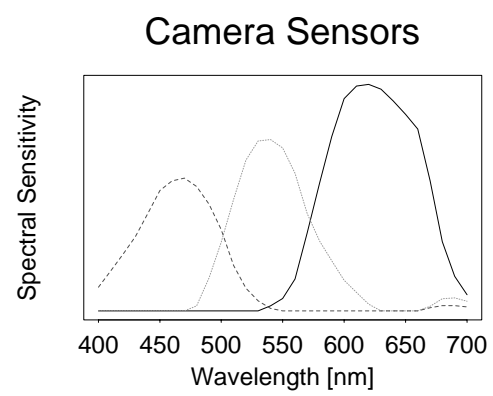

(b)
Sharpened Camera Sensors

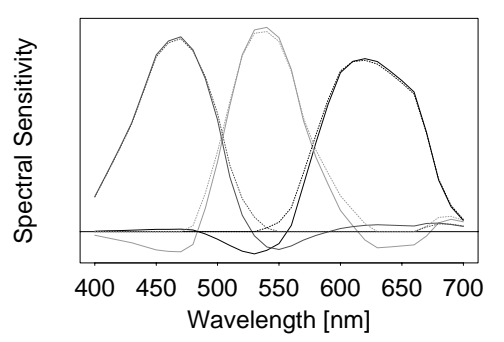

(c)

Fig. 2: 


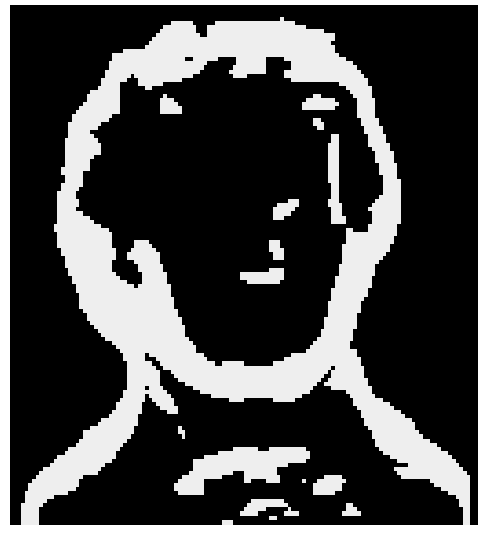

(a)

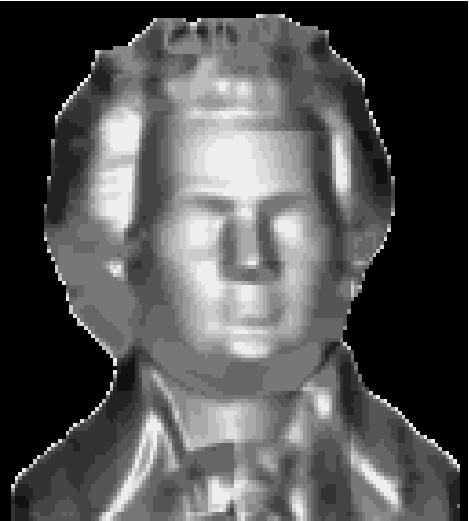

(a)

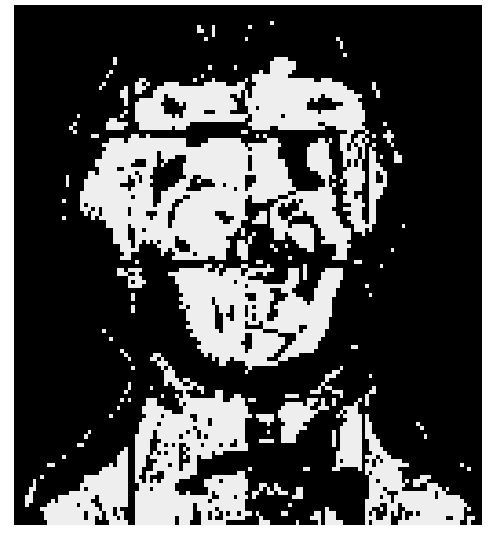

(b)

Fig. 3:

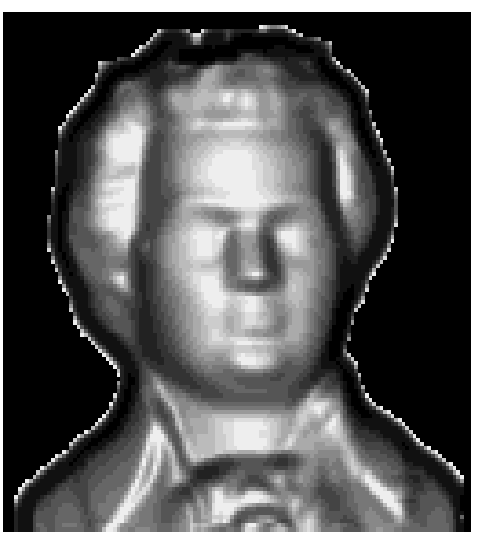

(b)

Fig. 4: 


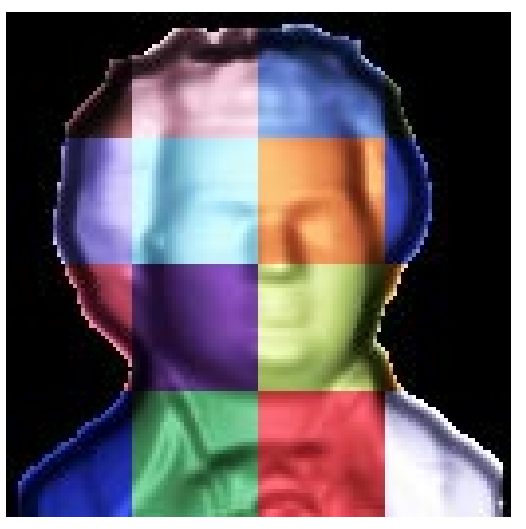

(a)

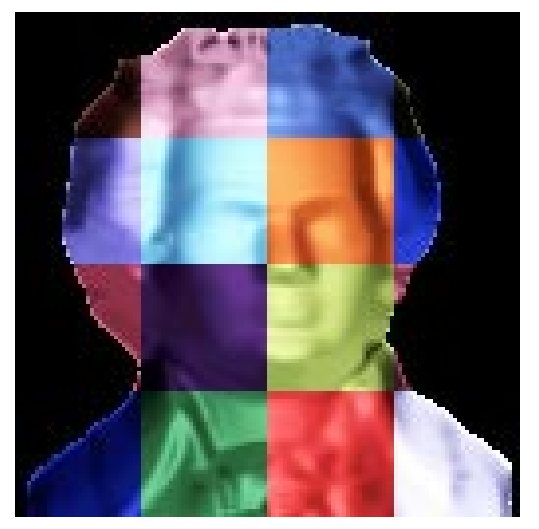

(b)

Fig. 5:

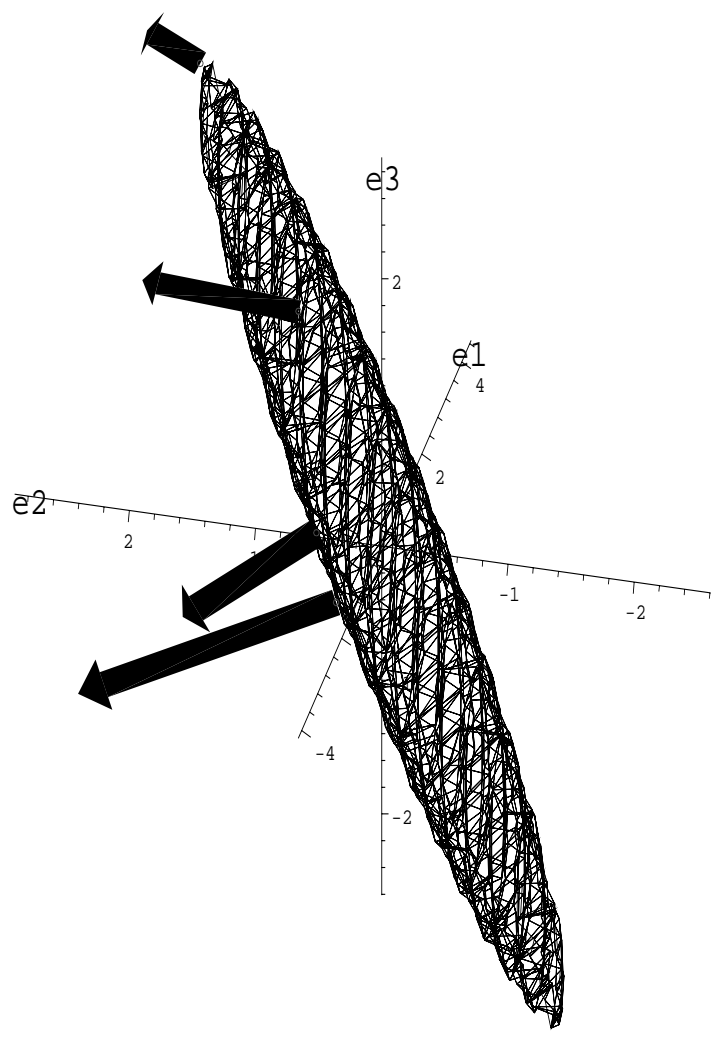

Fig. 6: 